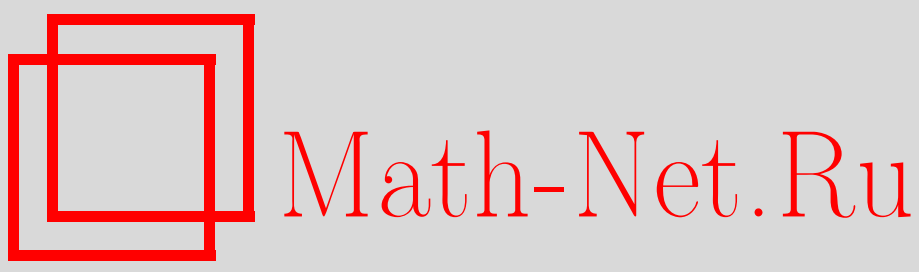

D. E. Pal'chunov, A. V. Trofimov, The theories of superatomic boolean algebras with distinguished subalgebra which lack countably saturated models, Sibirsk. Mat. Zh., 2020, Volume 61, Number 3, 654-668

DOI: https://doi.org/10.33048/smzh.2020.61.313

Use of the all-Russian mathematical portal Math-Net.Ru implies that you have read and agreed to these terms of use http://www . mathnet.ru/eng/agreement

Download details:

IP : 54.198 .64 .247

April 26, 2023, 04:34:43 
Сибирский математический журнал Май-июнь, 2020. Том 61, № 3

УДК 512.563

\title{
ТЕОРИИ СУПЕРАТОМНЫХ БУЛЕВЫХ АЛГЕБР С ВЫДЕЛЕННОЙ ПОДАЛГЕБРОЙ, НЕ ИМЕЮЩИЕ СЧЕТНО-НАСЫЩЕННОЙ МОДЕЛИ
}

\author{
Д. Е. Пальчунов, А. В. Трофимов
}

\begin{abstract}
Аннотация. Исследуются булевы алгебры с выделенной подалгеброй. Доказано существование континуума простых суператомных булевых алгебр с выделенной подалгеброй (подалгебра совпадает с булевой алгеброй по модулю идеала Фреше), элементарные теории которых различны и каждая из которых не имеет счетнонасыщенной модели.
\end{abstract}

DOI 10.33048/smzh.2020.61.313

Ключевые слова: булева алгебра, булева алгебра с выделенной подалгеброй, локальная алгебра, элементарная теория, конечно-аксиоматизируемая теория, разрешимая теория, элементарная эквивалентность.

\section{Введение}

Работа посвящена изучению теоретико-модельных свойств булевых алгебр с выделенной плотной подалгеброй конечной ширины. Одним из ключевых вопросов является наличие простых и счетно-насыщенных моделей у элементарных теорий. Простая модель - это счетная модель, в которой реализуются только главные типы [1] теории, счетно-насыщенная - счетная модель, в которой реализуются, наоборот, все типы, совместные с этой теорией. Другими словами, простая модель - это самая «маленькая» модель теории, счетнонасыщенная - самая «большая» модель из счетных моделей теории. Известно, что теория, имеющая счетно-насыщенную модель, имеет и простую модель.

Известно [2], что элементарная теория любой булевой алгебры имеет как простую, так и счетно-насыщенную модели. Если в сигнатуру булевых алгебр добавить одноместный предикат, выделяющий идеал, то ситуация с существованием насыщенных и простых моделей коренным образом меняется. В [3-9] исследовались булевы алгебры с выделенными идеалами, в частности, доказано, что существует континуум теорий булевых алгебр с одним выделенным идеалом, имеющих простую модель, но не имеющих счетно-насыщенной модели, существует континуум теорий, не имеющих простой модели, а также существует континуум элементарных теорий, имеющих счетно-насыщенную модель. Однако любая элементарная теория суператомной булевой алгебры с одним выделенным идеалом имеет простую и счетно-насыщенную модели $[3,4]$.

В настоящей работе доказано, что существует суператомная булева алгебра с выделенной плотной подалгеброй конечной ширины, теория которой не имеет счетно-насыщенной модели. В этой алгебре подалгебра изоморфна самой булевой алгебре, более того, подалгебра совпадает с булевой алгеброй по модулю идеала Фреше.

(с) 2020 Пальчунов Д. Е., Трофимов А. В. 


\section{1. Основные определения и предварительные результаты}

Будем рассматривать булевы алгебры в сигнатуре $\sigma=\langle\cup, \cap, C, 0,1\rangle$. Пусть $\sigma^{*}=\langle\sigma, P\rangle$, где $P$ - символ одноместного предиката, выделяющего подалгебру. Булеву алгебру с выделенной подалгеброй будем называть просто алгеброй. Через $P^{\mathfrak{A}}=\{a \in \mathfrak{A} \mid \mathfrak{A} \models P(a)\}$ обозначим основное множество выделенной подалгебры алгебры $\mathfrak{A}$.

Суператомную булеву алгебру с выделенной подалгеброй будем называть суператомной алгеброй. Пусть $\mathfrak{A}-$ счетная суператомная алгебра. Обозначим через $o(\mathfrak{A})$ наименьший ординал $\alpha$ такой, что единица алгебры принадлежит $(\alpha+1)$-му идеалу Фреше булевой алгебры, т. е. $1 \in F_{\alpha+1}(\mathfrak{A})$.

Пусть $\tau(\mathfrak{A})=(o(\mathfrak{A}), n)-$ тип суператомности [2] алгебры $\mathfrak{A}$, где $n-$ число атомов в фактор-алгебре $\mathfrak{A} / F_{\alpha}$ при $\alpha=o(\mathfrak{A})$.

Пусть $\mathfrak{A}$ - произвольная алгебра и $a \in P^{\mathfrak{A}}$ - элемент выделенной подалгебры. Определим алгебру, задаваемую элементом $a$ :

$(a)=\left\langle\{b \in \mathfrak{A} \mid b \leq a\}, \cup, \cap, C^{a}, 0, a, P^{\mathfrak{A}} \cap\{b \in \mathfrak{A} \mid b \leq a\}\right\rangle$, где $C^{a}(b)=a \cap C(b)$.

Прямая сумма $\sum_{i \in I} \mathfrak{A}_{i}$ определяется стандартным образом.

ОПРЕДЕЛЕНИЕ 1.1 [10]. Подалгебра $\mathfrak{B}$ булевой алгебры $\mathfrak{A}$ называется noдалгеброй ширины $n$, если под любым атомом подалгебры $\mathfrak{B}$ найдется не более $n$ атомов алгебры $\mathfrak{A}$, лежащих под ним, и любой атом алгебры $\mathfrak{A}$ лежит под некоторым атомом подалгебры $\mathfrak{B}$.

ОПРедЕЛЕНиЕ 1.2 [10]. Подалгебра $\mathfrak{B}$ булевой алгебры $\mathfrak{A}$ называется nлотной, если $\mathfrak{A}=\operatorname{sub}_{\mathfrak{A}}(\mathfrak{B}, F(\mathfrak{A}))$ - наименьшая подалгебра алгебры $\mathfrak{A}$, содержащая в себе подалгебру $\mathfrak{B}$ и идеал Фреше $F(\mathfrak{A})$.

Обозначим [10] через $K_{n}$ класс суператомных булевых алгебр с выделенной плотной подалгеброй ширины $n$.

ОПРЕДЕЛЕНИЕ 1.3 [10]. Обозначим $\mathfrak{A} \leq \mathfrak{B}$, если найдется $\mathfrak{C}$ такая, что $\mathfrak{B} \equiv \mathfrak{A} \times \mathfrak{C}$. Алгебраическая система $\mathfrak{A}$ называется неисчезающей, если из $\mathfrak{A} \equiv$ $\mathfrak{M} \times \mathfrak{N}$ следует $\mathfrak{A} \equiv \mathfrak{M}$ или $\mathfrak{A} \equiv \mathfrak{N}$.

ОПРЕДЕЛЕНИЕ 1.4 [10]. Алгебраическая система $\mathfrak{A}$ называется локалъной, если число попарно элементарно не эквивалентных неисчезающих алгебраических систем $\mathfrak{B} \leq \mathfrak{A}$ конечно, т. е. существуют $\mathfrak{B}_{1}, \ldots, \mathfrak{B}_{n}$ такие, что для произвольной неисчезающей $\mathfrak{C} \leq \mathfrak{A}$ выполнено $\mathfrak{C} \equiv \mathfrak{B}_{i}$ для некоторого $i \leq n$.

Следуя [10], приведем построение счетной последовательности формул $T_{k}(x), k \in \mathbb{N}$, языка $\sigma^{*}$ с одной свободной переменной.

Пусть $\varphi, \psi$ - формулы с одной свободной переменной языка $\sigma^{*}, T$ - теория класса булевых алгебр с выделенной подалгеброй. Обозначим

$\varphi \leq \psi$, если $T \vdash(\psi(x) \rightarrow(\exists y \leq x) \varphi(y)) ;$

$\varphi \# \psi$, если $T \vdash(\psi(x) \rightarrow(\forall y \leq x) \neg \varphi(y))$.

Определим отношение линейного порядка на конечных подмножествах множества натуральных чисел без нуля. Пусть $A=\left\{a_{1}>a_{2}>\cdots>a_{l}\right\}, B=\left\{b_{1}>\right.$ $\left.b_{2}>\cdots>b_{m}\right\} \subset \mathbb{N} \backslash\{0\}$ - два произвольных конечных подмножества множества натуральных чисел без нуля. Положим, $A<B$, если $\left(a_{1}, a_{2}, \ldots, a_{l}, 0, \ldots, 0\right)<$ $\left(b_{1}, b_{2}, \ldots, b_{m}, 0, \ldots, 0\right)$ в лексикографическом порядке, и $A \leq B$, если $A<B$ или $A=B$ (добавляем нули так, чтобы длины кортежей стали равными).

Обозначим $X=\{A \subset \mathbb{N} \backslash\{0\} \mid 2 \leq\|A\|<\omega\}$. Занумеруем множество $X$ в порядке возрастания. Пусть $\Omega: X \rightarrow \mathbb{N}$ - взаимно однозначное отображение всех конечных неодноэлементных подмножеств множества натуральных чисел 
без нуля на множество натуральных чисел такое, что $\Omega(A)<\Omega(B) \Longleftrightarrow A<$ $B$. Очевидно, что такое отображение можно определить так, чтобы оно было алгоритмически эффективным.

Зафиксируем $n \in \mathbb{N}$ и соответственно класс $K_{n}$. Перейдем к построению последовательности формул $T_{k}(x), k \in \mathbb{N}$ [10]. Построение будем вести по индукции.

БАЗис индУКции:

$T_{1}(x)=((x-$ атом $) \& P(x))$;

$T_{l}(x)=\left((x\right.$-объединение $l$ атомов $\left.) \& P(x) \&(\forall y \leq x) \bigwedge_{i<l} \neg T_{i}(y)\right)$ для $2 \leq l \leq n$.

При помощи формулы

$$
\begin{array}{r}
Q(x)=\exists y \exists z((x=y \cup z) \&(y \cap z=0) \&(\forall w \leq y)(w \neq 0 \rightarrow \neg P(w)) \\
\&(\forall w \leq z)(w \neq 0 \rightarrow \neg P(w)))
\end{array}
$$

для $2 \leq j \leq n$ определим

$$
\begin{array}{r}
T_{n+j-1}(x)=P(x) \&\left(\neg Q(x) \&(\exists y \leq x) T_{j}(y) \&\left(\bigwedge_{i \neq j, i \leq n}(\forall y \leq x) \neg T_{i}(y)\right)\right. \\
\&(\forall y, z \leq x)((y \cap z=0 \rightarrow(Q(y) \vee Q(z)))) .
\end{array}
$$

ШАГ индуКции. Предположим, что формулы $T_{1}(x), \ldots, T_{m}(x)$ построены. Пусть $R \in X, R \subset\{1,2, \ldots, m\}$, такое, что $T_{i} \# T_{j}$ для любых $i, j \in R, i \neq$ $j$, и имеющее наименьший номер $l=\Omega(R)$, который еще не был использован при построении формул $T_{1}(x), \ldots, T_{m}(x)$. Пусть $R=\left\{i_{1}, \ldots, i_{k}\right\}$. Определим множество $R^{*}$ и вспомогательные формулы $\pi_{R}(x), T_{R}(x)$ следующим образом:

$$
\begin{gathered}
R^{*}=\left\{i \leq m \mid\left(T_{i} \# T_{j}\right) \text { для всех } j \in R\right\} ; \\
\pi_{R}(x)=\left(\exists_{i \in R} x_{i}\right)\left(\bigwedge_{i \neq j}\left(x_{i} \cap x_{j}=0\right) \&\left(\bigcup_{i \in R} x_{i}=x\right) \&\left(\bigwedge_{i \neq j}\left(\forall z \leq x_{i}\right) \neg T_{j}(z)\right)\right),
\end{gathered}
$$

где $\left(\exists_{i \in R} x_{i}\right) \rightleftharpoons \exists x_{i_{1}} \ldots \exists x_{i_{k}}$;

$$
\begin{aligned}
& T_{R}(x)=\left(\neg \pi_{R}(x) \& P(x) \&(\forall y \leq x)\left(\left(\bigvee_{i \in R}(\forall z \leq y) \neg T_{i}(z)\right) \rightarrow \pi_{R}(y)\right)\right. \\
& \left.\quad \&\left((\forall y \leq x) \bigwedge_{i \in R^{*}} \neg T_{i}(y)\right) \&(\forall y \leq x)(\forall z \leq x)\left(y \cap z=0 \rightarrow\left(\pi_{R}(y) \vee \pi_{R}(z)\right)\right)\right) .
\end{aligned}
$$

Полагаем, $T_{m+1}(x)=T_{R}(x)$. Обозначим $R_{m+1}=R$ и $R_{m+1}^{*}=R^{*}$.

Для формул $T_{k}(x), n<k \leq 2 n-1$, обозначим:

$T_{R}(x)=T_{k}(x)$ для множества $R=\{k+1-n\} ; \quad R^{*}=\{i \leq n \mid i \neq k+1-n\}$.

ЗАмЕчАниЕ 1.5. Пусть $\mathfrak{A} \in K_{n}$. $1^{\mathfrak{A}}$ является атомом подалгебры $P(\mathfrak{A})$ тогда и только тогда, когда найдется число $l \leq n$ такое, что $\mathfrak{A} \models T_{l}(1)$.

ДокАЗАТЕЛЬство. В силу определения 1.1 найдется число $l \leq n$ такое, что $1^{\mathfrak{A}}$ - объединение $l$ атомов. Так как $1^{\mathfrak{A}}$ является атомом подалгебры, $\neg T_{1}(1), \ldots, T_{l-1}(1)$. Стало быть, $T_{l}(1)$.

Обратно, пусть $T_{l}(1)$. Предположим, что найдется $b<a$ такой, что $b$ является атомом подалгебры $P(\mathfrak{A})$. Тогда $T_{k}(b)$ для некоторого $k \leq l$. С другой стороны, $(\forall y \leq x) \neg T_{k}(y)$ в силу определения формулы $T_{l}$. Полученное противоречие завершает доказательство замечания. 
При помощи построенной последовательности формул для каждой алгебры $\mathfrak{A}$ и каждого элемента $a \in \mathfrak{A}$ определена характеристическая функция $w_{a}: \mathbb{N} \rightarrow$ $\mathbb{N}$ следующим образом:

$$
\begin{aligned}
& w_{a}(0)=0, \text { если } \mathfrak{A} \models P(a) ; \\
& w_{a}(0)=1, \text { если } \mathfrak{A} \models \neg P(a) ; \\
& w_{a}(m)=0, \text { если } \mathfrak{A} \models(\forall y \leq a) \neg T_{m}(y), m \geq 1 ; \\
& w_{a}(m)=\sup \left\{k \in \mathbb{N} \mid \mathfrak{A} \models\left(\exists x_{1}, \ldots, \exists x_{k} \leq a\right)\left(\left(\bigwedge_{i \neq j} x_{i} \cap x_{j}=0\right)\right.\right. \\
& \left.\left.\qquad \&\left(\bigwedge_{i \leq k} T_{m}\left(x_{i}\right)\right)\right)\right\}, m \geq 1, \text { иначе. }
\end{aligned}
$$

Положим $w_{\mathfrak{A}}=w_{1^{\mathfrak{A}}}$.

ОПРЕДЕЛЕНИЕ $1.6[10]$. Формула $\varphi(x)$ с одной свободной переменной сигнатуры $\sigma^{*}$ называется неисчезающей, если

$$
T \vdash(((x=y \cup z) \&(y \cap z=0) \& \varphi(x) \& P(y) \& P(z)) \rightarrow(\varphi(y) \vee \varphi(z))) .
$$

Предложение 1.7 [10]. Справедливы следующие утверждения:

(а) для любого числа $k \geq 1$ формула $T_{k}(x)$ неисчезающая;

(б) для любого числа $k \geq 1$ формула $T_{k}(1)$ полная в теории $T$, т. е. любые две модели теории $T$, на которых истинна формула $T_{k}(1)$, элементарно эквивалентны;

(в) если $i<j$, то $T_{j} \# T_{i}$;

(г) для любых $i, j$ либо $T_{i} \leq T_{j}$, либо $T_{i} \# T_{j}$.

Теорема 1.8 [10]. Пусть $\mathfrak{A}$ и $\mathfrak{B}$ - локальные булевы алгебры с выделенными подалгебрами, принадлежащими классу $K_{n}$. Для того чтобы $\mathfrak{A} \equiv \mathfrak{B}$, необходимо и достаточно, чтобы $w_{\mathfrak{A}}=w_{\mathfrak{B}}$.

Рассмотрим обогащение сигнатуры $\sigma_{1}=\sigma^{*} \cup\left\{c_{1}, \ldots, c_{k}\right\}$ конечным числом константных символов. Через $\left(\mathfrak{A}, a_{1}, \ldots, a_{k}\right)$ обозначим булеву алгебру с выделенной подалгеброй в обогащенной константами сигнатуре, где $a_{1}, \ldots, a_{k}-$ значения в модели $\mathfrak{A}$ константных символов $c_{1}, \ldots, c_{k}$ соответственно.

Пусть $\left(\mathfrak{A}, a_{1}, \ldots, a_{k}\right)$ и $\left(\mathfrak{B}, b_{1}, \ldots, b_{k}\right)$ - булевы алгебры с выделенными подалгебрами в обогащенной константами сигнатуре $\sigma_{1}, A_{0} \subseteq \mathfrak{A}$ и $B_{0} \subseteq \mathfrak{B}$. Отображение $f: A_{0} \rightarrow B_{0}$ назовем частичным изоморфизмом из $\left(\mathfrak{A}, a_{1}, \ldots, a_{k}\right)$ в $\left(\mathfrak{B}, b_{1}, \ldots, b_{k}\right)$, если множество $A_{0}$ определяет подалгебру в алгебре $\left(\mathfrak{A}, a_{1}, \ldots, a_{k}\right)$, множество $B_{0}-$ подалгебру в алгебре $\left(\mathfrak{B}, b_{1}, \ldots, b_{k}\right)$ (в частности $a_{1}, \ldots, a_{k} \in A_{0}$ и $\left.b_{1}, \ldots, b_{k} \in B_{0}\right)$ и отображение $f$ является изоморфизмом соответствующих подалгебр, т. е. для любых $a, b \in A_{0}$ выполнено

$$
\begin{gathered}
f(a \cup b)=f(a) \cup f(b) ; \quad f(a \cap b)=f(a) \cap f(b) ; \quad f(C(a))=C(f(a)) ; \\
P(a) \Longleftrightarrow P(f(a)) ; \quad f(0)=0 ; \quad f(1)=1 ; \quad f\left(a_{1}\right)=b_{1}, \ldots, f\left(a_{k}\right)=b_{k} .
\end{gathered}
$$

Обозначим $\operatorname{dom} f=A_{0}, \operatorname{im} f=B_{0}$.

Отображение $f: A_{0} \rightarrow B_{0}$ назовем конечным частичным изоморфизмом, если это отображение является частичным изоморфизмом и его область определения $\operatorname{dom} f=A_{0}$ конечна.

Говорят, что алгебра $\left(\mathfrak{A}, a_{1}, \ldots, a_{k}\right)$ m-эквивалентна алгебре $\left(\mathfrak{B}, b_{1}, \ldots, b_{k}\right)$, и используют обозначение $\left(\mathfrak{A}, a_{1}, \ldots, a_{k}\right) \equiv_{m}\left(\mathfrak{B}, b_{1}, \ldots, b_{k}\right)$, если существуют непустые множества $F_{0}, \ldots, F_{m}$ конечных частичных изоморфизмов из $\left(\mathfrak{A}, a_{1}\right.$, $\left.\ldots, a_{k}\right)$ в $\left(\mathfrak{B}, b_{1}, \ldots, b_{k}\right)$, удовлетворяющие следующему условию: для любых $l<m, f \in F_{l}, a \in \mathfrak{A}$ и $b \in \mathfrak{B}$ найдутся $g_{1}, g_{2} \in F_{l+1}$ такие, что $g_{1}, g_{2} \supset f$, $a \in \operatorname{dom} g_{1}$ и $b \in \operatorname{im} g_{2}$. 
Будем обозначать $\mathfrak{A} \equiv_{m} \mathfrak{B}$ для алгебр без выделенных констант, т. е. если $\sigma_{1}=\sigma^{*}$.

Пусть $\mathfrak{A}-$ произвольная булева алгебра с выделенной подалгеброй и $a \in \mathfrak{A}$. Введем обозначения:

$M(a) \rightleftharpoons\left\{m \neq 0 \mid w_{a}(m) \neq 0\right\}-$ носитель характеристической функции $w_{a} ;$

$N(a) \rightleftharpoons\left\{m \in M(a) \mid \forall n \in M(a)\left(T_{m} \leq T_{n} \rightarrow m=n\right)\right\}-$ множество максимальных элементов множества $M(a)$ относительно следующего порядка: $\leq(m, n) \Longleftrightarrow T_{m} \leq T_{n}$.

Обозначим $M(\mathfrak{A}) \rightleftharpoons M\left(1^{\mathfrak{A}}\right)$ и $N(\mathfrak{A}) \rightleftharpoons N\left(1^{\mathfrak{A}}\right)$.

Следующее определение является уточнением аналогичного определения из [10].

ОПРеДЕлЕниЕ 1.9. Элемент $a$ булевой алгебры с выделенной подалгеброй называется $m$-простым, если $N(a)=\{m\}$ и выполнено $P(a)$. Элемент $a$ называется простым, если $a \in F(\mathfrak{A})$ и найдутся число $l \leq n$ и $l$-простой элемент $d \geq a$ или $a$ является $m$-простым для некоторого $m$. Алгебра $\mathfrak{A}$ называется nростой (m-простой), если $1^{\mathfrak{A}}$ - простой (m-простой) элемент.

Предложение 1.10 [10]. Пусть $\mathfrak{A} \in K_{n}$ и $a \in \mathfrak{A}$. В алгебре $\mathfrak{A}$ выполнено $T_{l}(a)$ тогда и только тогда, когда элемент а является $l$-простым и $w_{a}(l)=1$.

Предложение 1.11 [10]. Пусть $\mathfrak{A}$ и $\mathfrak{B}-l$ - простые алгебры, принадлежащие классу $K_{n}$. Предположим, что $w_{\mathfrak{A}}(l), w_{\mathfrak{B}}(l) \geq 2^{m}$ и $l>n$. Тогда $\mathfrak{A} \equiv_{m} \mathfrak{B}$.

Предложение 1.12 [10]. Для того чтобы алгебра $\mathfrak{A}$ была локальной, необходимо и достаточно, чтобы множество $M(\mathfrak{A})$ было конечным.

Будем говорить, что элемент $a \in \mathfrak{A}$ локальный, если множество $M(a)$ конечно, и нелокальный в противном случае.

Предложение 1.13 [10]. Пусть $\mathfrak{A}$ - счетная локальная булева алгебра с выделенной подалгеброй, принадлежащая классу $K_{n}$. Тогда любой элемент алгебры $\mathfrak{A}$ есть объединение конечного числа попарно не пересекающихся простых элементов.

ОПРЕДЕЛЕниЕ 1.14 [10]. Пусть $\mathfrak{A}$ и $\mathfrak{B}$ - алгебры, принадлежащие классу $K_{n}$. Множество $S \subset \mathfrak{A} \times \mathfrak{B} \times \mathbb{N}$ называется признаком $m$-эквивалентности, если $S$ удовлетворяет следующим условиям:

Ax1) $\left(0^{\mathfrak{A}}, 0^{\mathfrak{B}}, 0\right),\left(1^{\mathfrak{A}}, 1^{\mathfrak{B}}, 0\right) \in S$

$\mathrm{Ax} 2)(a, b, k) \in S, k<m \rightarrow(a, b, k+1) \in S$;

$\mathrm{Ax} 3)(a, b, k) \in S \rightarrow((a=0 \Longleftrightarrow b=0) \& P(a) \& P(b) \& k \leq m)$;

$\mathrm{Ax} 4)$ если $(a, b, k) \in S, k<m$ и $0<c<a$, то найдутся попарно не пересекающиеся $c_{0}, \ldots, c_{l} \leq a$, попарно не пересекающиеся $d_{1}, \ldots, d_{l} \leq b$ и число $j \leq l$ такие, что $\bigcup_{i \leq l} c_{i}=a, \bigcup_{i \leq l} d_{i}=b, c_{1} \cup \cdots \cup c_{j}=c,\left(c_{i}, d_{i}, k+1\right) \in S$ при $i \geq 2, c_{0} \cup c_{1} \in F(\mathfrak{A})$ и $\left(c_{0} \cup c_{1}\right) \cong\left(d_{1}\right)$ (здесь и в дальнейшем имеется в виду изоморфизм булевых алгебр с выделенными подалгебрами);

$\mathrm{Ax} 5)$ если $(a, b, k) \in S, k<m$ и $0<d<b$, то найдутся попарно непересекающиеся $d_{0}, \ldots, d_{l} \leq b$, попарно не пересекающиеся $c_{1}, \ldots, c_{l} \leq a$ и число $j \leq l$ такие, что $\bigcup_{i \leq l} c_{i}=a, \bigcup_{i \leq l} d_{i}=b, d_{1} \cup \cdots \cup d_{j}=d,\left(c_{i}, d_{i}, k+1\right) \in S$ при $i \geq 2$, $d_{0} \cup d_{1} \in F(\mathfrak{B})$ и $\left(d_{0} \cup d_{1}\right) \cong\left(c_{1}\right)$.

Предложение 1.15 [10]. Пусть $\mathfrak{A} \in K_{n}$ и $2 \leq i \leq n$. Тогда 
(a) если $w_{\mathfrak{A}}(i)=\infty$, то существует число $j>i$ такое, что $T_{i} \leq T_{j}$ и $w_{\mathfrak{A}}(j) \neq$ 0

(б) если $\mathfrak{A} i$-простая, то $w_{\mathfrak{A}}(i)<\infty$.

Теорема 1.16 [10]. Пусть алгебры $\mathfrak{A}$ и $\mathfrak{B}$ принадлежат классу $K_{n}$ и для них существует признак $m$-эквивалентности $S$. Тогда $\mathfrak{A} \equiv_{m} \mathfrak{B}$.

\section{2. Теории, не имеющие счетно-насыщенной модели}

ОПРЕДЕЛЕНИЕ 2.1 [1]. Счетная модель $\mathfrak{A}$ называется счетно-насыщенной, если для любых элементов $a_{1}, \ldots, a_{k} \in \mathfrak{A}$ любой тип формул $p\left(x, a_{1}, \ldots, a_{k}\right)$, совместный с теорией $\operatorname{Th}\left(\mathfrak{A}, a_{1}, \ldots, a_{k}\right)$, реализуется в модели $\left(\mathfrak{A}, a_{1}, \ldots, a_{k}\right)$, т. е. найдется элемент $a \in \mathfrak{A}$ такой, что для любой формулы $\varphi \in p\left(x, a_{1}, \ldots, a_{k}\right)$ выполнено $\left(\mathfrak{A}, a_{1}, \ldots, a_{k}\right) \models \varphi\left(a, a_{1}, \ldots, a_{k}\right)$.

Предложение 2.2. Пусть $\mathfrak{A}$ - алгебра, принадлежащая классу $K_{n}$. Предположим, что найдется бесконечное множество $X \subset M(\mathfrak{A})$ такое, что для всех $i, j \in X, i \neq j$, выполнено $T_{i} \# T_{j}$. Тогда элементарная теория Th $\mathfrak{A}$ не имеет счетно-насыщенной модели.

ДокАЗАТЕЛЬСтво. Пусть алгебра $\mathfrak{A}$ и множество $X \subset M(\mathfrak{A})$ удовлетворяют условиям предложения. Пусть $Y \subset X$. Рассмотрим следующее множество формул:

$$
\Delta_{Y}=\operatorname{Th}(\mathfrak{A}) \cup\left\{(\exists y \leq x) T_{i}(y) \mid i \in Y\right\} \cup\left\{\neg(\exists y \leq x) T_{i}(y) \mid i \in X \backslash Y\right\} .
$$

Докажем, что множество $\Delta_{Y}$ локально совместно. Зафиксируем множество $Y$ и обозначим $\Delta \rightleftharpoons \Delta_{Y}, \Delta^{1} \rightleftharpoons\left\{(\exists y \leq x) T_{i}(y) \mid i \in Y\right\}, \Delta^{2} \rightleftharpoons\left\{\neg(\exists y \leq x) T_{i}(y) \mid i \in\right.$ $X \backslash Y\}$. При этих обозначениях $\Delta=\operatorname{Th}(\mathfrak{A}) \cup \Delta^{1} \cup \Delta^{2}$.

Пусть $\Delta_{0}$ - конечное подмножество $\Delta, \Delta_{0}^{1}=\Delta_{0} \cap \Delta^{1}, \Delta_{0}^{2}=\Delta_{0} \cap \Delta^{2}$. Тогда $\Delta_{0}=\Delta_{0}^{1} \cup \Delta_{0}^{2}$ и $\Delta_{0}^{1} \cap \Delta_{0}^{2}=\varnothing$. Пусть $Y_{1}=\left\{i_{1}, \ldots, i_{m}\right\} \subset Y \subset M(\mathfrak{A})$ и $\Delta_{0}^{1}=\{(\exists y \leq$ $\left.x) T_{i}(y) \mid i \in Y_{1}\right\}$. Найдутся $c_{1}, \ldots, c_{m} \in \mathfrak{A}$ такие, что $\mathfrak{A} \models T_{i_{j}}\left(c_{j}\right)$ для всех $j \leq m$. Обозначим $a_{1}=c_{1}, a_{2}=c_{2} \backslash a_{1}, \ldots, a_{m}=c_{m} \backslash\left(a_{1} \cup \cdots \cup a_{m-1}\right)$. Тогда для $i, j \leq m$, $i \neq j$, выполнено $a_{i} \cap a_{j}=0$. Кроме того, в силу неисчезаемости формулы $T_{i_{j}}$ и того, что $T_{i} \# T_{j}$ для всех $i \neq j, i, j \in Y_{0}$, будет выполнено $\mathfrak{A} \models T_{i_{j}}\left(a_{j}\right)$ для любого $j \leq m$. Рассмотрим $a=a_{1} \cup \cdots \cup a_{m}$. Очевидно, что тогда $(\exists y \leq a) T_{k}(y)$ для любого $k \in Y_{1}$.

Пусть $Y_{2}=\left\{i \mid \neg(\exists y \leq x) T_{i}(y) \in \Delta_{0}^{2}\right\}$ и $k \in Y_{2}, b \leq a$ и выполнено $T_{k}(b)$. Тогда $b=\left(b \cap a_{1}\right) \cup \cdots \cup\left(\left(b \cap a_{m}\right)\right)$, причем $\left(b \cap a_{i}\right) \cap\left(b \cap a_{j}\right)=0$ при $i \neq j$. Следовательно, в силу того, что формула $T_{k}$ неисчезающая, выполнено $\mathfrak{A} \models T_{k}\left(b \cap a_{l}\right)$ для некоторого $l \leq m$, при этом $\mathfrak{A} \models T_{i_{l}}\left(a_{l}\right), i_{l} \in Y$, и $k \in Y_{2} \subset X \backslash Y$, значит, $i_{l} \neq k, i_{l}, k \in X$. Но тогда $T_{k} \# T_{i_{l}} ;$ противоречие с $\mathfrak{A} \models T_{k}\left(b \cap a_{l}\right), b \cap a_{l} \leq a_{l}$ и $\mathfrak{A} \models T_{i_{l}}\left(a_{l}\right)$. Следовательно, $\mathfrak{A} \models \neg(\exists y \leq b) T_{k}(y)$ для любого $k \in Y_{2}$. Значит, $\mathfrak{A} \models \Delta_{0}(a)$. Мы показали, что множество формул $\Delta_{Y}$ локально совместно. В силу локальной теоремы Мальцева множество $\Delta_{Y}$ совместное. Пусть $P_{Y}$ - максимальное непротиворечивое расширение множества $\Delta_{Y}$. Поскольку $\operatorname{Th}(\mathfrak{A}) \subset P_{Y}$, тип $P_{Y}$ совместен с теорией $\operatorname{Th}(\mathfrak{A})$. Так как различным подмножествам множества $X$ соответствуют различные типы, существует континуум типов, совместных с элементарной теорией $\mathrm{Th}(\mathfrak{A})$. Следовательно, элементарная теория Th $\mathfrak{A}$ не имеет счетно-насыщенной модели. Предложение доказано.

В [10] доказано следующее 
Предложение 2.3 [10]. (а) Для $n \geq 3$ найдется бесконечное множество $A \subset \mathbb{N}$ такое, что $T_{i} \# T_{j}$ для различных $i, j \in A$.

(б) Для любого числа $k \in \mathfrak{N}$ найдется алгебра $\mathfrak{N}_{k} \in K_{n}$ такая, что $\mathfrak{N}_{k} \models$ $T_{k}(1)$.

Теорема 2.4. Для любого $n \geq 3$ существует континуум суператомных булевых алгебр с выделенными плотными подалгебрами ширины $n$, элементарные теории которых различны и не имеют счетно-насыщенных моделей.

ДоказАтельство. Рассмотрим бесконечное множество $A \subset \mathbb{N}$ такое, что $T_{i} \# T_{j}$ для различных $i, j \in A$. Для бесконечного множества $I \subset A$ обозначим $\mathfrak{A}_{I}=\sum_{i \in I} \mathfrak{N}_{i}$. Тогда $I \subset M\left(\mathfrak{A}_{I}\right)$. В силу предложения 2.2 элементарная теория $\operatorname{Th} \mathfrak{A}_{I}$ не имеет счетно-насыщенной модели.

Пусть $I, J \subset A$ и $I \neq J$. Пусть, например, $I \not \subset J$. Тогда найдется число $i \in I \backslash J$. В этом случае $\mathfrak{A}_{I} \models \exists x T_{i}(x)$. Предположим, что $\mathfrak{A}_{J} \models \exists x T_{i}(x)$. Тогда найдется элемент $a \in \mathfrak{A}_{J}$ такой, что $T_{i}(a)$. Значит, $a \leq 1^{\mathfrak{N}_{j_{1}}} \cup \cdots \cup 1^{\mathfrak{N}_{j_{k}}}$ для некоторых $j_{1}, \ldots, j_{k} \in J$. В силу неисчезаемости формулы $T_{i}$ заключаем, что для некоторого $s \leq k$ выполнено $T_{i} \leq T_{j_{s}}$; противоречие. Стало быть, $\mathfrak{A}_{J} \models \neg \exists x T_{i}(x)$ и $\mathfrak{A}_{I} \not \equiv \mathfrak{A}_{J}$. Теорема доказана.

\section{3. Простые модели}

Напомним, что счетная модель $\mathfrak{A}$ простая, если в ней реализуются только главные типы. В настоящем разделе докажем, что все модели вида $\mathfrak{A}_{I}$, построенные в теореме 2.4, простые.

Предложение 3.1. Пусть $\mathfrak{A}_{1} \times \mathfrak{A}_{2} \equiv \mathfrak{B}_{1} \times \mathfrak{B}_{2}, w_{\mathfrak{A}_{2}}=w_{\mathfrak{B}_{2}}, \mathfrak{A}_{1}$ и $\mathfrak{B}_{1}$ локальные алгебры. Тогда $\mathfrak{A}_{2} \equiv \mathfrak{B}_{2}$.

ДокАЗАТЕЛЬСтво. Если $\mathfrak{A}_{2}$ и $\mathfrak{B}_{2}$ локальные, то доказывать нечего. Пусть $\mathfrak{A}_{2}$ и $\mathfrak{B}_{2}$ нелокальные. Зафиксируем $m \in \mathbb{N}$. Докажем, что $\mathfrak{A}_{2} \equiv_{m} \mathfrak{B}_{2}$. Определим

$$
\begin{gathered}
M=M\left(\mathfrak{A}_{1} \times \mathfrak{B}_{1}\right) \cup\{1,2, \ldots, n\} ; \\
U=\left\{\exists z\left(P(z) \&(M(z) \subseteq M) \&\left(w_{C(z)}(l)=0\right)\right) \mid l \in M\right\} \\
\cup\left\{w_{\mathbf{1}}(l)=k \mid l \in M, k \leq 2^{m}\right\} \cup\left\{w_{\mathbf{1}}(l) \geq 2^{m} \mid l \in M\right\} \cup\{P(x)\} .
\end{gathered}
$$

Возьмем число $t^{\prime}$ такое, что все формулы из семейства $U$ устойчивы относительно $t^{\prime}$-эквивалентности, и определим $t=t^{\prime}+m$. Построим признак $S$ $m$-эквивалентности алгебр $\mathfrak{A}_{2}$ и $\mathfrak{B}_{2}$ :

$S=\left\{(x, y, k) \mid x \in \mathfrak{A}_{2}, y \in \mathfrak{B}_{2}\right.$, и выполнены следующие условия:

(1) $k \leq m, P(x), P(y)$;

(2) $\forall l \in M\left(w_{x}(l)=w_{y}(l)\right.$ или $\left.w_{x}(l), w_{y}(l) \geq 2^{m-k}\right)$;

(3) $(x) \times \mathfrak{C} \equiv_{t-k}(y) \times \mathfrak{D}$ для некоторых локальных $\mathfrak{C}, \mathfrak{D} \in K_{n}, M(\mathfrak{C} \times \mathfrak{D}) \subseteq M$;

(4) $\left.(\forall l \leq n)\left(l \neq 1 \rightarrow w_{x}(l)=w_{y}(l)\right)\right\}$.

Покажем, что множество $S$ удовлетворяет требованиям Ax1-Ax5 из определения 1.14 . $S$

Ax1. В силу условий настоящего утверждения $\left(0^{\mathfrak{A}_{2}}, 0^{\mathfrak{B}_{2}}, 0\right),\left(1^{\mathfrak{A}_{2}}, 1^{\mathfrak{B}_{2}}, 0\right) \in$

Aх2. По построению множества $S$ если $(x, y, k) \in S$, то $(x, y, k+1) \in S$.

Ах3. Пусть $(x, y, k) \in S$ и $x=0$. Тогда для любого $i \leq n$ выполнено $0=w_{x}(i)=w_{y}(i)$. Следовательно, $y=0$. 
Ах4. Пусть $(x, y, k) \in S, k<m, 0<a<x$.

Случай 1: выполнено $P(a)$. Поскольку $(x) \times \mathfrak{C} \equiv_{t-k}(y) \times \mathfrak{D}$, найдутся элемент $0<b<y$ и разложения $\mathfrak{C}=\mathfrak{C}_{1} \times \mathfrak{C}_{2}, \mathfrak{D}=\mathfrak{D}_{1} \times \mathfrak{D}_{2}$ такие, что

$$
(a) \times \mathfrak{C}_{1} \equiv_{t-(k+1)}(b) \times \mathfrak{D}_{1} ; \quad(x \backslash a) \times \mathfrak{C}_{2} \equiv_{t-(k+1)}(y \backslash b) \times \mathfrak{D}_{2} .
$$

Множество всех элементов $b<y, b>0$, удовлетворяющих выше указанным условиям, обозначим через $H$. Пусть $b \in H$.

Заметим, что тогда для $(a, b, k+1)$ и $(x \backslash a, y \backslash b, k+1)$ выполнены следующие условия признака $S$ :

(1) $P(b)$ и $P(y \backslash b)$ в силу того, что $P(x)$ принадлежит $U$ и, следовательно, устойчиво относительно $t-(k+1)$-эквивалентности.

(3) $(a) \times \mathfrak{C}_{1} \equiv_{t-(k+1)}(b) \times \mathfrak{D}_{1}, M\left(\mathfrak{C}_{1} \times \mathfrak{D}_{1}\right) \subseteq M$ и

$(x \backslash a) \times \mathfrak{C}_{2} \equiv_{t-(k+1)}(y \backslash b) \times \mathfrak{D}_{2}, M\left(\mathfrak{C}_{2} \times \mathfrak{D}_{2}\right) \subseteq M$.

Покажем, что найдется элемент $b \in H$ такой, что выполнено условие (2) признака $S$, а именно, $\forall l \in M\left(w_{a}(l)=w_{b}(l)\right.$ или $\left.w_{a}(l), w_{b}(l) \geq 2^{m-(k+1)}\right)$ и $\forall l \in M\left(w_{x \backslash a}(l)=w_{y \backslash b}(l)\right.$ или $\left.w_{x \backslash a}(l), w_{x \backslash a}(l) \geq 2^{m-(k+1)}\right)$.

Предположим, что такого элемента $b \in H$ не существует. Тогда для каждого $b \in H$ обозначим $l_{b}=\max \{l \in M \mid$ условие (2) признака $S$ нарушается на $l$ для $(a, b, k+1)$ или $(x \backslash a, y \backslash b, k+1)\}$.

Рассмотрим элемент $b \in H$ с наименьшим значением $l_{b}$. Обозначим $l=l_{b}$. Без ограничения общности можно считать, что условие $S$ нарушается для $l$ на $(a, b, k+1)$. Возможны следующие случаи.

Случай 1.1: $w_{b}(l)<w_{a}(l), w_{b}(l)<2^{m-(k+1)}, w_{x}(l), w_{y}(l) \geq 2^{m-k}, w_{x \backslash a}(l)<$ $2^{m-(k+1)}, l>n$ или $l=1$. В частности, рассматривается в том числе случай, когда $w_{a}(l)=\infty$.

Тогда $w_{a}(l)=w_{x}(l)-w_{x \backslash a}(l) \geq 2^{m-(k+1)}$ и $w_{y \backslash b}(l) \geq 2^{m-(k+1)}$. Так как $w_{x \backslash a}(l)<2^{m-(k+1)}$, имеем

$$
\left.(x \backslash a) \times \mathfrak{C}_{2} \models \exists z\left(P(z) \& M(z) \subseteq M \& w_{C(z)}(l)=0\right)\right) .
$$

Стало быть, в силу устойчивости множества формул $U$ относительно, $t^{\prime}$-эквивалентности, заключаем, что $\left.(y \backslash b) \times \mathfrak{D}_{2} \models \exists z\left(P(z) \& M(z) \subseteq M \& w_{C(z)}(l)=0\right)\right)$. Значит, найдется локальный элемент $c \leq y \backslash b$, принадлежащий подалгебре, такой, что $w_{y \backslash(b \cup c)}(l)=0$ и $M(c) \subset M$. Докажем, что найдется $l$-простой элемент $d \leq c$ такой, что $w_{c \backslash d}(l)=0$. Предположим противное. Тогда в силу классификации локальных алгебр найдется $s$-простой элемент $c_{1} \leq c$ такой, что $T_{l} \leq T_{s}$, $l<s$. Следовательно, $(y \backslash b) \models T_{s}\left(c_{1}\right)$ и $w_{y \backslash b}(s) \geq 1$. Так как $w_{x \backslash a}(l)<2^{m-(k+1)}$, то $w_{x \backslash a}(s)=0$. Поскольку $M(c) \subseteq M$, то $s \in M$. Таким образом,

$$
0=w_{x \backslash a}(s)<w_{y \backslash b}(s), \quad s \in M, s>l_{b}=l .
$$

Противоречие с тем, что $l=l_{b}-$ наибольшее число, для которого нарушается условие (2) признака $S$. Следовательно, существует $l$-простой элемент $d \leq c$ такой, что $w_{c \backslash d}(l)=0$. Возьмем $l$-простой элемент $d_{1} \leq d$ такой, что $w_{d_{1}}(l)=$ $w_{x \backslash a}(l)$. Определим $b^{*}=b \cup\left(d \backslash d_{1}\right)$. Тогда

$$
\begin{gathered}
w_{x \backslash a}(l)=w_{d_{1}}(l)=w_{y \backslash b^{*}}(l), \quad w_{a}(l) \geq 2^{m-(k+1)}, \\
w_{b^{*}}(l)=w_{y}(l)-w_{y \backslash b^{*}}(l)=w_{y}(l)-w_{x \backslash a}(l) \geq 2^{m-(k+1)},
\end{gathered}
$$

так как $w_{y}(l) \geq 2^{m-k}$ и $w_{x \backslash a}(l)<2^{m-(k+1)}$. 
Поскольку $(a) \times \mathfrak{C}_{1} \equiv_{t-(k+1)}(b) \times \mathfrak{D}_{1}$, то
$(a) \times\left(d \backslash d_{1}\right) \times \mathfrak{C}_{1} \equiv_{t-(k+1)}\left(b^{*}\right) \times \mathfrak{D}_{1}, \quad M\left(\mathfrak{C}_{1} \times \mathfrak{D}_{1} \times\left(d \backslash d_{1}\right)\right) \subseteq M$.

Аналогично

$(x \backslash a) \times \mathfrak{C}_{2} \equiv_{t-(k+1)}\left(y \backslash b^{*}\right) \times \mathfrak{D}_{2} \times\left(d \backslash d_{1}\right), \quad M\left(\mathfrak{C}_{2} \times \mathfrak{D}_{2} \times\left(d \backslash d_{1}\right)\right) \subseteq M$.

Таким образом, условие (2) признака $S$ выполняется для $l$ на $\left(a, b^{*}, k+1\right),(x \backslash$ $\left.a, y \backslash b^{*}, k+1\right)$, при этом условие (2) признака $S$ выполнено для всех $l^{\prime}>l$ на $\left(a, b^{*}, k+1\right),\left(x \backslash a, y \backslash b^{*}, k+1\right)$ автоматически. Следовательно, $l_{b^{*}}<l$. Противоречие с выбором элемента $b$ и числа $l$.

СлучАй $1.2: w_{b}(l)<w_{a}(l), w_{b}(l)<2^{m-(k+1)}, w_{x}(l), w_{y}(l) \geq 2^{m-k}, w_{x \backslash a}(l) \geq$ $2^{m-(k+1)}, l>n$ или $l=1$.

Тогда найдется $l$-простой элемент $c \leq y \backslash b$ с характеристикой

$$
w_{c}(l)=\min \left\{2^{m-(k+1)}-w_{b}(l), w_{a}(l)-w_{b}(l)\right\} .
$$

В этом случае полагаем $b^{*}=b \cup c$. Следовательно, либо $w_{a}(l)=w_{b^{*}}(l)$, либо $w_{a}(l), w_{b^{*}}(l) \geq 2^{m-(k+1)}$. При этом $w_{x \backslash a}(l), w_{y \backslash b^{*}}(l) \geq 2^{m-(k+1)}$.

Из $(a) \times \mathfrak{C}_{1} \equiv_{t-(k+1)}(b) \times \mathfrak{D}_{1}$ следуют $(a) \times(c) \times \mathfrak{C}_{1} \equiv_{t-(k+1)}\left(b^{*}\right) \times \mathfrak{D}_{1}$ и $M\left(\mathfrak{C}_{1} \times \mathfrak{D}_{1} \times(c)\right) \subseteq M$.

Аналогично

$$
(x \backslash a) \times \mathfrak{C}_{2} \equiv_{t-(k+1)}\left(y \backslash b^{*}\right) \times(c) \times \mathfrak{D}_{2}, \quad M\left(\mathfrak{C}_{2} \times \mathfrak{D}_{2} \times(c)\right) \subseteq M .
$$

Таким образом, условие (2) признака $S$ выполняется для $l$ на $\left(a, b^{*}, k+1\right)$, $\left(x \backslash a, y \backslash b^{*}, k+1\right)$; противоречие с выбором элемента $b$ и числа $l$.

СлУчАЙ 1.3: $w_{b}(l)<w_{a}(l), w_{x}(l)=w_{y}(l)<2^{m-k}, l>n$ или $l=1$.

Так как $w_{x}(l)=w_{y}(l)<\infty$, то $w_{a}(l), w_{b}(l), w_{x \backslash a}(l), w_{y \backslash b}(l)<\infty$. Стало быть, найдется $l$-простой элемент $c \leq y \backslash b$ с характеристикой $w_{c}(l)=w_{a}(l)-w_{b}(l)$. Определив $b^{*}=b \cup c$, получим

$$
(a) \times(c) \times \mathfrak{C}_{1} \equiv_{t-(k+1)}\left(b^{*}\right) \times \mathfrak{D}_{1}
$$

и

$M\left(\mathfrak{C}_{1} \times \mathfrak{D}_{1} \times(c)\right) \subseteq M ;(x \backslash a) \times \mathfrak{C}_{2} \equiv_{t-(k+1)}\left(y \backslash b^{*}\right) \times(c) \times \mathfrak{D}_{2}, M\left(\mathfrak{C}_{2} \times \mathfrak{D}_{2} \times(c)\right) \subseteq M ;$

$w_{a}(l)=w_{b^{*}}(l)$ и $w_{x \backslash a}(l)=w_{y \backslash b^{*}}(l)$.

Таким образом, условие (2) признака $S$ выполняется для $l$ на $\left(a, b^{*}, k+1\right)$, $\left(x \backslash a, y \backslash b^{*}, k+1\right)$; противоречие с выбором элемента $b$ и числа $l$.

СлучАй 1.4: $w_{a}(l)<w_{b}(l), l>n$ или $l=1$. Тогда $w_{a}(l)<2^{m-(k+1)}$, так как в противном случае условие (2) признака $S$ выполнено для $l$ для $(a, b, k+1)$. С учетом рассуждений, аналогичных проведенным для случая 1.1 , существует $l$-простой элемент $c \leq b$ такой, что $w_{b \backslash c}(l)=0$. Возьмем $l$-простой $c_{1} \leq c$ такой, что $w_{c_{1}}(l)=w_{a}(l)$. Положим $b^{*}=(b \backslash c) \cup c_{1}$. Тогда $b=b^{*} \cup\left(c \backslash c_{1}\right)$ и справедливо следующее:

$$
\begin{array}{cc}
w_{b^{*}}(l)=w_{a}(l) ; \quad & w_{x \backslash a}(l)=w_{y \backslash b^{*}}(l) \text { либо } w_{x \backslash a}(l), \quad w_{y \backslash b^{*}}(l) \geq 2^{m-(k+1)} ; \\
& (a) \times \mathfrak{C}_{1} \equiv_{t-(k+1)}\left(b^{*}\right) \times\left(c \backslash c_{1}\right) \times \mathfrak{D}_{1}
\end{array}
$$

и

$$
M\left(\mathfrak{C}_{1} \times \mathfrak{D}_{1} \times\left(c \backslash c_{1}\right)\right) \subseteq M ; \quad(x \backslash a) \times\left(c \backslash c_{1}\right) \times \mathfrak{C}_{2} \equiv_{t-(k+1)}\left(y \backslash b^{*}\right) \times \mathfrak{D}_{2},
$$


и

$$
M\left(\mathfrak{C}_{2} \times \mathfrak{D}_{2} \times\left(c \backslash c_{1}\right)\right) \subseteq M
$$

Отсюда следует, что условие (2) признака $S$ выполняется для $l$ на $\left(a, b^{*}, k+1\right)$, $\left(x \backslash a, y \backslash b^{*}, k+1\right)$; противоречие с выбором элемента $b$ и числа $l$.

СлУчАй 1.5: $w_{a}(l)=w_{b}(l), w_{x \backslash a}(l) \neq w_{y \backslash b}(l), l>n$ или $l=1$. Разбирается аналогично.

СлучАЙ 1.6: $1<l \leq n$. Если $w_{a}(l)=\infty$, то найдется $l^{\prime}>l$ такое, что $T_{l}<T_{l^{\prime}}$ и $w_{a}\left(l^{\prime}\right) \neq 0$. В таком случае $w_{b}\left(l^{\prime}\right) \neq 0$. Следовательно, $w_{b}(l)=\infty$. Аналогично $w_{x \backslash a}(l)=\infty \Longleftrightarrow w_{y \backslash b}(l)=\infty$.

Пусть $w_{b}(l)<w_{a}(l)<\infty$. Поскольку $w_{x}(l)=w_{y}(l)$, найдется $l$-простой элемент $c \leq y \backslash b$ такой, что $w_{c}(l)=w_{a}(l)-w_{b}(l)$. Положим $b^{*}=b \cup c$. Тогда

$$
w_{a}(l)=w_{b^{*}}(l) ; \quad w_{x \backslash a}(l)=w_{y \backslash b^{*}}(l) ; \quad(a) \times(c) \times \mathfrak{C}_{1} \equiv_{t-(k+1)}\left(b^{*}\right) \times \mathfrak{D}_{1}
$$

и

$$
M\left(\mathfrak{C}_{1} \times \mathfrak{D}_{1} \times(c)\right) \subseteq M ; \quad(x \backslash a) \times \mathfrak{C}_{2} \equiv_{t-(k+1)}\left(y \backslash b^{*}\right) \times(c) \times \mathfrak{D}_{2},
$$

и

$$
M\left(\mathfrak{C}_{2} \times \mathfrak{D}_{2} \times(c)\right) \subseteq M .
$$

В этом случае условие $(2)$ признака $S$ выполняется для $l$ на $\left(a, b^{*}, k+1\right),(x \backslash$ $\left.a, y \backslash b^{*}, k+1\right)$.

Итак, доказано, существование $0<b<y$ такого, что выполнены условия (1)-(3) признака $S$.

Докажем существование $b^{*}$ такого, что выполнены все условия (1)-(4) признака $S$ для $\left(a, b^{*}, k+1\right)$ и $\left(x \backslash a, y \backslash b^{*}, k+1\right)$. Предположим, что для некоторого числа $1<l \leq n$ нарушается условие (4) признака $S$ : $w_{a}(l) \neq w_{b}(l)$ или $w_{x \backslash a}(l) \neq w_{y \backslash b}(l)$. Пусть для определенности $w_{a}(l)<w_{b}(l)$. Заметим, что $w_{a}(l)<\infty \Longleftrightarrow w_{b}(l)<\infty$. В самом деле, пусть $w_{a}(l)<\infty$. В силу устойчивости множества формул $U$ относительно $t^{\prime}$-эквивалентности, практически дословно повторяя доказательство из случая 1.1 , заключаем, что найдется $l$ простой элемент $b_{1} \leq b$ такой, что $w_{b \backslash b_{1}}(l)=0$. Стало быть, $w_{b_{1}}(l)=w_{b}(l)$. Следовательно, в силу предложения $1.15 w_{b}(l)<\infty$. Аналогично $w_{x \backslash a}(l)<$ $\infty \Longleftrightarrow w_{y \backslash b}(l)<\infty$. Стало быть, найдется $l$-простой элемент $c \leq b$ с характеристикой $w_{c}(l)=w_{b}(l)-w_{a}(l)$. Полагаем $b^{*}=b \backslash c$. Так как $w_{x}(l)=w_{y}(l)$, имеем

$$
w_{a}(l)=w_{b^{*}}(l), w_{x \backslash a}(l)=w_{y \backslash b^{*}}(l) ; \quad(a) \times(c) \times \mathfrak{C}_{1} \equiv_{t-(k+1)}\left(b^{*}\right) \times \mathfrak{D}_{1}
$$

и

$$
M\left(\mathfrak{C}_{1} \times \mathfrak{D}_{1} \times(c)\right) \subseteq M ; \quad(x \backslash a) \times \mathfrak{C}_{2} \equiv_{t-(k+1)}\left(y \backslash b^{*}\right) \times(c) \times \mathfrak{D}_{2},
$$

и

$$
M\left(\mathfrak{C}_{2} \times \mathfrak{D}_{2} \times(c)\right) \subseteq M .
$$

Изменяя элемент $b$ вышеуказанным образом для всех $1<l \leq n$, на которых нарушается условие (4) признака $S$, получим элемент $b^{*}$ такой, что будут выполнены все условия (1)-(4) признака $S$ для $\left(a, b^{*}, k+1\right)$ и $\left(x \backslash a, y \backslash b^{*}, k+1\right)$.

Таким образом, если $P(a)$, то существование требуемого числа $b$ доказано.

СлучАй 2: $\neg P(a)$. Тогда найдутся непересекающиеся элементы $a_{1}, a_{2}, a_{3}$, $a_{4} \leq x$ такие, что выполнены следующие условия:

$x=\left(a_{1} \cup a_{2}\right) \cup\left(a_{3} \cup a_{4}\right), P\left(a_{1}\right), P\left(a_{4}\right), a_{2} \in F\left(\mathfrak{A}_{2}\right), a_{3} \in F\left(\mathfrak{A}_{2}\right)$;

$\left(\forall e \leq a_{2}\right)(e \neq 0 \rightarrow \neg P(e)),\left(\forall e \leq a_{3}\right)(e \neq 0 \rightarrow \neg P(e))$; 
$a=a_{1} \cup a_{2}$.

Так как $P\left(a_{1}\right)$ и $(x, y, k) \in S$, аналогично рассмотренному случаю 1 найдутся элементы $b_{1}, b_{2} \leq y$ такие, что $b=b_{1} \cup b_{2}, P\left(b_{1}\right), P\left(b_{2}\right), b_{1} \cap b_{2}=0$ и при этом $\left(a_{1}, b_{1}, k+1\right) \in S,\left(a_{2} \cup a_{3} \cup a_{4}, b_{2}, k+1\right) \in S$.

Поскольку $\left(a_{2} \cup a_{3} \cup a_{4}, b_{2}, k+1\right) \in S$, то $w_{a_{2} \cup a_{3} \cup a_{4}}(i)=w_{b_{2}}(i)$ для всех $1<i \leq n$. Следовательно, найдется элемент $b_{3} \leq b_{2}$ такой, что $\left(b_{3}\right) \cong\left(a_{2} \cup a_{3}\right)$.

Докажем, что $\left(a_{4}, b_{2} \backslash b_{3}, k+1\right) \in S$, а именно проверим последовательно выполнение условий (1)-(4).

(1) Выполнено очевидным образом;

(2) Пусть $l \in M$. Так как $w_{a_{2} \cup a_{3}}(l)=w_{b_{3}}(l)<\infty$, то $w_{a_{4}}(l)=w_{a_{2} \cup a_{3} \cup a_{4}}(l)-$ $w_{a_{2} \cup a_{3}}(l)$ и $w_{b_{2} \backslash b_{3}}(l)=w_{b_{2}}(l)-w_{b_{3}}(l)$. Заметим, что $M\left(a_{2} \cup a_{3}\right)=M\left(b_{3}\right) \subseteq$ $\{2,3, \ldots, n\}$. Следовательно, для $l>n$ или $l=1$ выполнено $w_{a_{4}}(l)=w_{a_{2} \cup a_{3} \cup a_{4}}(l)$ и $w_{b_{2} \backslash b_{3}}(l)=w_{b_{2}}(l)$. Так как $\left(a_{2} \cup a_{3} \cup a_{4}, b_{2}, k+1\right) \in S$, либо $w_{a_{4}}(l), w_{b_{2} \backslash b_{3}}(l) \geq$ $2^{m-(k+1)}$, либо $w_{a_{4}}(l)=w_{b_{2} \backslash b_{3}}(l)$. Случай $l \leq n, l \neq 1$, будет рассмотрен при доказательстве (4).

(3) $\left(a_{4}\right) \times\left(a_{2} \cup a_{3}\right) \times \mathfrak{C}_{1} \equiv\left(a_{2} \cup a_{3} \cup a_{4}\right) \times \mathfrak{C}_{1} \equiv_{t-(k+1)}\left(b_{2}\right) \times \mathfrak{D}_{1} \equiv\left(b_{2} \backslash b_{3}\right) \times$ $\left(b_{3}\right) \times \mathfrak{D}_{1}$ и $M\left(\left(a_{2} \cup a_{3}\right) \times \mathfrak{C}_{1} \times \mathfrak{D}_{1}\right) \subseteq M$.

(4) Пусть $i \leq n$ и $i \neq 1$. Тогда

$w_{a_{4}}(i)=w_{a_{2} \cup a_{3} \cup a_{4}}(i)-w_{a_{2} \cup a_{3}}(i)=w_{b_{2}}(i)-w_{b_{3}}(i)=w_{b_{2} \backslash b_{3}}(i)$.

Следовательно, $\left(a_{4}, b_{2} \backslash b_{3}, k+1\right) \in S$.

Таким образом, доказано, что множество $S$ является признаком $m$-эквивалентности и, стало быть, $\mathfrak{A}_{2} \equiv \mathfrak{B}_{2}$.

Предложение доказано.

ЗАмечАниЕ 3.2. Пусть $\mathfrak{A} \in K_{n}$. Для каждого элемента $a \in \mathfrak{A}$ найдутся попарно не пересекающиеся элементы $c, d_{1}, \ldots, d_{k} \leq a$ такие, что $a=c \cup d_{1} \cup$ $\cdots \cup d_{k}, P(c), d_{1}, \ldots, d_{k} \in A t(\mathfrak{A})$ и $\left(\forall z \leq d_{1} \cup \cdots \cup d_{k}\right)(P(z) \rightarrow z=0)$. Набор элементов $c, d_{1}, \ldots, d_{k}$ является однозначным с точностью до их перестановки.

ДокАЗАтЕльство. Пусть $a \in \mathfrak{A}$. Так как $\mathfrak{A} \in K_{n}$, найдутся элементы $c_{1}, c_{2} \leq a$ такие, что $a=c_{1} \cup c_{2}, P\left(c_{1}\right), c_{2} \in F(\mathfrak{A})$ и $c_{1} \cap c_{2}=0$. Пусть $D=\{d \mid$ $\left.d \leq c_{2}, P(d)\right\}$. Обозначим $c \rightleftharpoons c_{1} \cup \bigcup_{d_{\in} D} d$. Тогда $a=c \cup(a \backslash c)$ и $(\forall z \leq a \backslash c)(P(z) \rightarrow$ $z=0)$. Поскольку $a \backslash c \in F(\mathfrak{A})$, найдутся непересекающиеся атомы $d_{1}, \ldots, d_{k}$ такие, что $a \backslash c=d_{1} \cup \cdots \cup d_{k}$. Элементы $c, d_{1}, \ldots, d_{k}$ искомые.

Докажем однозначность. Заметим, что для каждого элемента $d_{i}$ не существует элемента $e \geq d_{i}$ такого, что $P(e)$ и $e \leq a$. Стало быть, для двух различных разложений $a=c \cup d_{1} \cup \cdots \cup d_{k}, a=c^{1} \cup d_{1}^{1} \cup \cdots \cup d_{k^{1}}^{1}$, удовлетворяющих требуемым свойствам, для каждого $i \leq k$ выполнено $d_{i} \leq d_{1}^{1} \cup \cdots \cup d_{k^{1}}^{1}$. Следовательно, $d_{1} \cup \cdots \cup d_{k}=d_{1}^{1} \cup \cdots \cup d_{k^{1}}^{1}$ и $c=c^{1}$.

ОПРЕДЕЛЕНИЕ 3.3. Пусть $\mathfrak{A} \in K_{n}$ и элементы $a, c, d_{1} \ldots, d_{k} \in \mathfrak{A}$ удовлетворяют требованиям замечания 3.2. Обозначим $f(a)=c, E(a)=\left\{d_{1}, \ldots, d_{k}\right\}$, $e(a)=d_{1} \cup \cdots \cup d_{k}, a^{1}=a, a^{0}=C(a)$. Для каждого $d \in E(a)$ через $g(d)$ обозначим атом подалгебры $P^{\mathfrak{A}}$, лежащий над $d$, т. е. $d \leq g(d)$ и $g(d) \in A t\left(P^{\mathfrak{A}}\right)$.

Теорема 3.4. Пусть каждое разложение $\mathfrak{A} \equiv \mathfrak{B} \times \mathfrak{C}$ счетной алгебры $\mathfrak{A} \in$ $K_{n}$ удовлетворяет следующим условиям:

(a) если $\mathfrak{B}$ локальна, то для любого $l \in N(\mathfrak{B})$ если $w_{\mathfrak{B}}(l)=\infty$, то $w_{\mathfrak{C}}(l)<\infty$;

(б) если $\mathfrak{B}$ нелокальна, то $\mathfrak{C}$ локальна.

Тогда $\mathfrak{A}$ - простая модель. 
ДокАЗАтельство. Рассмотрим случай, когда $\mathfrak{A}$ нелокальна. Пусть $b_{1}, \ldots, b_{k} \in \mathfrak{A}$. Докажем существование полной формулы $\varphi\left(x_{1}, \ldots, x_{k}\right)$ такой, что $\mathfrak{A} \models \varphi\left(b_{1}, \ldots, b_{k}\right)$. Отсюда и будет следовать, что модель $\mathfrak{A}$ простая.

Пусть $r=2^{k}-1,\{0,1\}^{k}=\left\{\epsilon_{0}, \ldots, \epsilon_{r}\right\}$. Определим $a_{i}=b^{\epsilon_{i}}, i \leq r$. Тогда $a_{i} \cap$ $a_{j}=0$ для $i \neq j, i, j \leq r$ и $a_{0} \cup \cdots \cup a_{r}=1$. Для каждого $i \leq r$ справедливо $a_{i}=$ $f\left(a_{i}\right) \cup e\left(a_{i}\right)$. Следовательно, $1=f\left(a_{0}\right) \cup \cdots \cup f\left(a_{r}\right) \cup\left(e\left(a_{0}\right) \cup \cdots \cup e\left(a_{r}\right)\right)$, причем $P\left(e\left(a_{0}\right) \cup \cdots \cup e\left(a_{r}\right)\right)$. В силу условия (б) без ограничения общности можно считать, что $f\left(a_{0}\right)$ нелокальный. Тогда $f\left(a_{1}\right), \ldots, f\left(a_{r}\right)$ локальные. Заметим, по условию (а), если $l \in N\left(f\left(a_{i}\right)\right)$ и $w_{f\left(a_{i}\right)}(l)=\infty$ для $i \geq 1$, то $w_{f\left(a_{j}\right)}(l)<\infty$ для любого $j \neq i, j \leq r$.

Обозначим

$$
\begin{gathered}
L_{i}=\left\{j \in N\left(f\left(a_{i}\right)\right) \mid w_{f\left(a_{i}\right)}(j)<\infty\right\}, \quad i \geq 1 ; \\
M=\bigcup_{i \geq 1} M\left(f\left(a_{i}\right)\right) ; \quad M_{0}=M \cup M\left(e\left(a_{0}\right) \cup \cdots \cup e\left(a_{r}\right)\right) ; \\
L_{0}=\left\{j \in M \mid w_{f\left(a_{0}\right)}(j)<\infty\right\}, \\
\psi\left(x_{0}, \ldots, x_{r}\right)=\left(\bigwedge_{i \geq 1} \bigwedge_{j \in L_{i}}\left(w_{f\left(x_{i}\right)}(j)=w_{f\left(a_{i}\right)}(j)\right)\right) \&\left(\bigwedge_{i \geq 1}\left(M\left(f\left(x_{i}\right)\right)=M\left(f\left(a_{i}\right)\right)\right)\right) \\
\left.\&\left(\bigwedge_{j \in L_{0}}\left(w_{f\left(x_{0}\right)}(j)=w_{f\left(a_{0}\right)}(j)\right)\right) \&\left(\bigwedge_{j \in M_{0}}\left(w_{f\left(a_{0}\right)}(j) \neq 0 \Longleftrightarrow w_{f\left(x_{0}\right)}(j) \neq 0\right)\right)\right) \\
\&\left(\bigwedge_{I \subseteq\{0, \ldots, r\}}\left(w \bigcup_{i \rightarrow n I} e\left(x_{i}\right)=w_{i \in I} e\left(a_{i}\right)\right)\right) .
\end{gathered}
$$

Рассмотрим формулу $\varphi\left(y_{1}, \ldots, y_{k}\right)$, полученную из формулы $\psi$ подстановкой терма $y^{\epsilon_{i}}$ вместо $x_{i}$. Очевидно, что $\mathfrak{A}=\varphi\left(b_{1}, \ldots, b_{k}\right)$. Докажем, что построенная формула полная. Пусть

$$
\left(\mathfrak{B}, c_{1}, \ldots, c_{k}\right) \models \operatorname{Th}(\mathfrak{A}) \cup\left\{\phi\left(c_{1}, \ldots, c_{k}\right)\right\} .
$$

Докажем, что $\left(\mathfrak{B}, c_{1}, \ldots, c_{k}\right) \equiv\left(\mathfrak{A}, b_{1}, \ldots, b_{k}\right)$.

Пусть $d_{i}=c^{\epsilon_{i}}, i \leq r$. Тогда $\left(\mathfrak{B}, d_{0}, \ldots, d_{r}\right) \models \psi\left(d_{0}, \ldots, d_{r}\right)$. Заметим, что $d_{i} \cap d_{j}=0$ для $i \neq j, i, j \leq r$ и $d_{0} \cup \cdots \cup d_{r}=1$. Поскольку $M\left(f\left(d_{i}\right)\right)=M\left(f\left(a_{i}\right)\right)$ для $i \geq 1$, элементы $f\left(d_{1}\right), \ldots, f\left(d_{r}\right)$ локальны. Поскольку $\mathfrak{B}$ нелокальна, $f\left(d_{0}\right)$ нелокальный.

Докажем, что $w_{f\left(a_{i}\right)}=w_{f\left(d_{i}\right)}$. Пусть $i \geq 1$. Тогда в силу построения формулы $\psi$ заключаем, что $M\left(f\left(a_{i}\right)\right)=M\left(f\left(d_{i}\right)\right)$. Достаточно доказать, что для всех $j \in N\left(f\left(a_{i}\right)\right)=N\left(f\left(d_{i}\right)\right)$ имеет место $w_{f\left(a_{i}\right)}(j)=w_{f\left(d_{i}\right)}(j)$. Возьмем произвольное $j \in M\left(f\left(a_{i}\right)\right)=M\left(f\left(d_{i}\right)\right)$. Если $w_{f\left(a_{i}\right)}(j)<\infty$, то $j \in L_{i}$ и $w_{f\left(a_{i}\right)}(j)=w_{f\left(d_{i}\right)}(j)$. Пусть $w_{f\left(a_{i}\right)}(j)=\infty$. Предположим, что $w_{f\left(d_{i}\right)}(j)<\infty$. Тогда $w_{f\left(a_{l}\right)}(j)<\infty$ для всех $l \neq i, l \leq r$. Значит, $j \in L_{l}$ для всех $l \neq i, l \leq r$. Следовательно, $w_{f\left(d_{l}\right)}(j)<\infty$ для всех $l \leq r$. Последнее влечет $w_{\mathfrak{B}}(j)<\infty$; противоречие с $\mathfrak{A} \equiv \mathfrak{B}$. Полученное противоречие доказывает, что $w_{f\left(a_{i}\right)}=w_{f\left(d_{i}\right)}$. Следовательно, $\left(f\left(a_{i}\right)\right) \equiv\left(f\left(d_{i}\right)\right)$ для $i \geq 1$.

Докажем, что $w_{f\left(a_{0}\right)}=w_{f\left(d_{0}\right)}$. Пусть $j \notin M_{0}$. Тогда $w_{f\left(a_{0}\right)}(j)=w_{\mathfrak{B}}(j)=$ $w_{\mathfrak{A}}(j)=w_{f\left(d_{0}\right)}$. Зафиксируем $j \in M_{0}$. Тогда $w_{f\left(a_{0}\right)}(j) \neq 0 \Longleftrightarrow w_{f\left(d_{0}\right)}(j) \neq$ 0. Стало быть, $M\left(f\left(a_{0}\right)\right)=M\left(f\left(d_{0}\right)\right)$. Рассмотрим $j \in N\left(f\left(a_{0}\right)\right.$. Возможны следующие два случая. 
СлучАй 1: $j \in M$. Если $w_{f\left(a_{0}\right)}(j)<\infty$, то $j \in L_{0}$ и $w_{f\left(a_{0}\right)}(j)=w_{f\left(d_{0}\right)}(j)$. Пусть $w_{f\left(a_{0}\right)}(j)=\infty$. В этом случае $w_{f\left(a_{i}\right)}(j)<\infty$ для всех $i \leq r, i \neq 0$. Следовательно,

$$
\begin{aligned}
w_{f\left(a_{0}\right)}(j) & =w_{\mathfrak{A}}(j)-w_{f\left(a_{1}\right)}(j)-\cdots-w_{f\left(a_{r}\right)}(j)-w_{e\left(a_{0}\right) \cup \cdots \cup e\left(a_{r}\right)}(j) \\
& =w_{\mathfrak{B}}(j)-w_{f\left(d_{1}\right)}(j)-\cdots-w_{f\left(d_{r}\right)}(j)-w_{e\left(d_{0}\right) \cup \cdots \cup e\left(d_{r}\right)}(j)=w_{f\left(d_{0}\right)}(j) .
\end{aligned}
$$

Случай 2: $j \in M_{0} \backslash M$. Тогда

$$
w_{f\left(a_{0}\right)}(j)=w_{\mathfrak{A}}(j)-w_{e\left(a_{0}\right) \cup \cdots \cup e\left(a_{r}\right)}(j)=w_{\mathfrak{B}}(j)-w_{e\left(d_{0}\right) \cup \cdots \cup e\left(d_{r}\right)}(j)=w_{f\left(d_{0}\right)}(j) .
$$

Таким образом, $w_{f\left(a_{0}\right)}=w_{f\left(d_{0}\right)}$. Так как

$$
\left(\bigwedge_{I \subset\{0, \ldots, r\}}\left(w_{i \in I} e\left(x_{i}\right)=w_{i \in I} e\left(a_{i}\right)\right)\right)
$$

имеем

$$
\left(e\left(a_{0}\right) \cup \cdots \cup e\left(a_{r}\right), e\left(a_{0}\right), \ldots, e\left(a_{r}\right)\right) \cong\left(e\left(d_{0}\right) \cup \cdots \cup e\left(d_{r}\right), e\left(d_{0}\right), \ldots, e\left(d_{r}\right)\right) .
$$

Обозначим $\mathfrak{M}_{1}=\left(f\left(a_{1}\right)\right) \times \cdots \times\left(f\left(a_{r}\right)\right) \times\left(e\left(a_{0}\right) \cup \cdots \cup e\left(a_{r}\right)\right), \mathfrak{M}_{2}=\left(f\left(d_{1}\right)\right) \times$ $\cdots \times\left(f\left(d_{r}\right)\right) \times\left(e\left(d_{0}\right) \cup \cdots \cup e\left(d_{r}\right)\right)$. Суммируя вышеизложенное, имеем

$$
\left(f\left(a_{0}\right)\right) \times \mathfrak{M}_{1} \equiv\left(f\left(d_{0}\right)\right) \times \mathfrak{M}_{2} ; \mathfrak{M}_{1} \equiv \mathfrak{M}_{2} ;
$$

$$
\mathfrak{M}_{1}, \mathfrak{M}_{2} \text { - локальные алгебры; } w_{f\left(a_{0}\right)}=w_{f\left(d_{0}\right)} \text {. }
$$

Отсюда в силу предложения 3.1 заключаем, что $\left(f\left(a_{0}\right)\right) \equiv\left(f\left(d_{0}\right)\right)$.

Далее

$$
\begin{aligned}
&\left(\mathfrak{A}, a_{0}, \ldots, a_{r}\right) \equiv((\left.\left.f\left(a_{0}\right)\right), f\left(a_{0}\right), 0, \ldots, 0\right) \\
& \times\left(\left(f\left(a_{1}\right)\right)\right.\left., 0, f\left(a_{1}\right), 0, \ldots, 0\right) \times \cdots \times\left(\left(f\left(a_{r}\right)\right), 0, \ldots, 0, f\left(a_{r}\right)\right) \\
& \times\left(\left(e\left(a_{0}\right) \cup \cdots \cup e\left(a_{r}\right)\right), e\left(a_{1}\right), \ldots, e\left(a_{r}\right)\right) \\
& \equiv\left(\left(f\left(d_{1}\right)\right), 0, f\left(d_{1}\right), 0, \ldots, 0\right) \times \cdots \times\left(\left(f\left(d_{r}\right)\right), 0, \ldots, 0, f\left(d_{r}\right)\right) \\
& \times\left(\left(e\left(d_{0}\right) \cup \cdots \cup e\left(d_{r}\right)\right), e\left(d_{1}\right), \ldots, e\left(d_{r}\right)\right) \\
& \\
& \quad \times\left(\left(f\left(d_{0}\right)\right), f\left(d_{0}\right), 0, \ldots, 0\right) \equiv\left(\mathfrak{B}, d_{0}, \ldots, d_{r}\right) .
\end{aligned}
$$

Заметим, что $b_{1}, \ldots, b_{k}$ термально выражаются через $a_{1}, \ldots, a_{r}$ точно так же, как $c_{1}, \ldots, c_{k}$ через $d_{1}, \ldots, d_{r}$. Следовательно, $\left(\mathfrak{A}, b_{1}, \ldots, b_{k}\right) \equiv\left(\mathfrak{B}, c_{1}, \ldots, c_{k}\right)$ и, стало быть, формула $\phi$ полная.

Случай, когда $\mathfrak{A}$ локальна, разбирается аналогично.

Теорема доказана.

Следуя [10], для каждого $k \in \mathbb{N}$ построим $\mathfrak{N}_{k}$ такую, что $\mathfrak{N}_{k} \models T_{k}(1)$. Построение будем вести индукцией по числу $k$.

БАзис индукции: $k \leq n$. В этом случае $\mathfrak{N}_{k}$ есть $k$-атомная булева алгебра с выделенной двухэлементной подалгеброй.

Пусть $n<k \leq 2 n-1$ и $j=k+1-n$. Тогда $\mathfrak{N}_{k}=\mathfrak{N}_{j}^{\omega}$.

ШАГ индукции. Предположим, что для всех $l<k$ алгебра $\mathfrak{N}_{l}$ построена. Для формулы $T_{k}$ найдется непустое не одноэлементное конечное множество $R \subset$ $\mathbb{N}$ такое, что $T_{k}=T_{R}$. Пусть для определенности $R=\left\{l_{1}, \ldots, l_{m}\right\}$. Обозначим $\mathfrak{N}_{k}=\left(\mathfrak{N}_{l_{1}} \times \cdots \times \mathfrak{N}_{l_{m}}\right)^{\omega}$. 
Предложение 3.5. Для любого $k \in \mathbb{N}$ модель $\mathfrak{N}_{k}$ простая.

ДокАЗАТЕльство. Требуемое утверждение докажем индукцией по $k$. Докажем, что любое разложение алгебры $\mathfrak{N}_{k}=\mathfrak{B} \times \mathfrak{C}$ удовлетворяет требованию (а) из теоремы 3.4 .

БАзис индукции. Если $k \leq n$, то алгебра $\mathfrak{N}_{k}$ простая и $w_{\mathfrak{N}_{k}}(l)=0$ для всех $l \neq k$.

Пусть $n<k \leq 2 n-1$ и $j=k+1-n$. В этом случае $\mathfrak{N}_{k}=\mathfrak{N}_{j}^{\omega}$. Пусть $\mathfrak{N}_{k}=\mathfrak{B} \times \mathfrak{C}, l \in N(\mathfrak{B})$ и $w_{\mathfrak{B}}(l)=\infty$. В этом случае $l=j$ и для любого числа $i \in \mathbb{N}$ выполнено $\mathfrak{B} \not \leq \mathfrak{N}_{j}^{i}$. Стало быть, найдется число $i \in \mathbb{N}$ такое, что $\mathfrak{C} \leq \mathfrak{N}_{j}^{i}$. Последнее влечет, что $w_{\mathfrak{C}}(j)=w_{\mathfrak{C}}(l) \leq k<\infty$. Следовательно, разложение алгебры $\mathfrak{N}_{k}=\mathfrak{B} \times \mathfrak{C}$ удовлетворяет требованию (а) из теоремы 3.4 , поэтому алгебра $\mathfrak{N}_{k}$ простая.

ШАГ индукции. Пусть $k>2 n-1$. Тогда найдется конечное не одноэлементное множество $R=\left\{n_{1}, \ldots, n_{s}\right\} \subset \mathbb{N}$ такое, что $T_{k}=T_{R}$. В силу индукционного предположения алгебры $\mathfrak{N}_{n_{1}}, \ldots, \mathfrak{N}_{n_{s}}$ простые. Пусть $\mathfrak{M}=\mathfrak{N}_{n_{1}} \times \cdots \times \mathfrak{N}_{n_{s}}$. Тогда $\mathfrak{N}_{k}=\mathfrak{M}^{\omega}$.

Пусть $\mathfrak{N}_{k}=\mathfrak{B} \times \mathfrak{C}, l \in N(\mathfrak{B})$ и $w_{\mathfrak{B}}(l)=\infty$. Предположим, что $\mathfrak{B} \leq \mathfrak{M}^{r}$ для некоторого $r \in \mathbb{N}$. Так как $\mathfrak{B}$ локальная, она является прямой суммой конечного числа неисчезающих алгебр: $\mathfrak{B}=\mathfrak{B}_{1} \times \cdots \times \mathfrak{B}_{m}, \mathfrak{B}_{i}-$ неисчезающие алгебры. Пусть для определенности $\mathfrak{B}_{1}-l$-простая алгебра и $w_{\mathfrak{B}_{1}}(l)=\infty$. Так как $\mathfrak{B}_{1}$ неисчезающая, без ограничения общности можно считать, что $\mathfrak{B}_{1} \leq \mathfrak{N}_{n_{1}}$. Тогда найдется алгебра $\mathfrak{C}_{1} \leq \mathfrak{N}_{n_{1}}$ такая, что $\mathfrak{N}_{n_{1}}=\mathfrak{B}_{1} \times \mathfrak{C}_{1}$. Так как $\mathfrak{N}_{n_{1}}$ простая, указанное разложение в силу индукционного предположения удовлетворяет требованию (а) из теоремы 3.4. Стало быть, $w_{\mathfrak{C}_{1}}(l)<\infty$. Следовательно, $\mathfrak{N}_{n_{1}} \equiv \mathfrak{B}_{1}$, $l=n_{1}$ и $w_{\mathfrak{N}_{n_{1}}}\left(n_{1}\right)=\infty$; противоречие с $\mathfrak{N}_{n_{1}}=T_{n_{1}}(1)$. Следовательно, $\mathfrak{B} \not \leq \mathfrak{M}^{r}$ для любого $r \in \mathbb{N}$. Стало быть, $\mathfrak{B} \models \exists x T_{k}(x)$ и $w_{\mathfrak{B}}(k)=1<\infty$.

Таким образом, любое разложение удовлетворяет требованиям (а) из теоремы 3.4.

Предложение доказано.

Следствие 3.6. Для любого $k \in \mathbb{N}$ модель $\mathfrak{N}_{k}^{\omega}$ простая.

ДокАЗАТЕЛЬСТво. Пусть $\mathfrak{N}_{k}^{\omega}=\mathfrak{B} \times \mathfrak{C}, l \in N(\mathfrak{B})$ и $w_{\mathfrak{B}}(l)=\infty$. Тогда найдется $l$-простая алгебра $\mathfrak{B}_{1} \leq \mathfrak{B}$ такая, что $w_{\mathfrak{B}_{1}}(l)=\infty$. Предположим, что $\mathfrak{B} \leq \mathfrak{N}_{k}^{r}$ для некоторого $r \in \mathbb{N}$. Так как $\mathfrak{N}_{k}$ простая и неисчезающая, $\mathfrak{N}_{k} \equiv \mathfrak{B}_{1}$. Значит, $l=k$ и $w_{\mathfrak{N}_{k}}(k)=\infty$. Полученное противоречие доказывает, что $\mathfrak{C} \leq \mathfrak{N}_{k}^{r}$ для некоторого $r \in \mathbb{N}$. Следовательно, $w_{\mathfrak{C}}(l) \leq r<\infty$.

Предложение доказано.

Следствие 3.7. Теория любой локальной алгебры имеет простую модель.

ДокАЗАТЕЛьство. Пусть $\mathfrak{A}-$ локальная алгебра и $N(\mathfrak{A})=\left\{k_{1}, \ldots, k_{s}\right\}$. Тогда найдутся простые неисчезающие модели $\mathfrak{B}_{1}, \ldots, \mathfrak{B}_{s}$ такие, что $\mathfrak{A} \equiv \mathfrak{B}_{1} \times$ $\cdots \times \mathfrak{B}_{s}$ и $\mathfrak{B}_{i} k_{i}$-простая для всех $i \leq s$.

Пусть $\mathfrak{A}=\mathfrak{B} \times \mathfrak{C}, l \in N(\mathfrak{B})$ и $w_{\mathfrak{B}}(l)=\infty$. Тогда найдется $l$-простая алгебра $\mathfrak{D} \leq \mathfrak{B}$ такая, что $w_{\mathfrak{D}}(l)=\infty$. В этом случае для некоторого $i \leq s$ будет выполнено $\mathfrak{D} \equiv \mathfrak{B}_{i}$. Так как $T_{k_{i}} \# T_{k_{j}}$ для $i, j \leq s, i \neq j$, то $w_{\mathfrak{D}}\left(k_{j}\right)=0$. Следовательно, модель $\mathfrak{B}_{1} \times \cdots \times \mathfrak{B}_{s}$ простая.

Следствие доказано. 
Теорема 3.8. Для любого $n \geq 3$ существует континуум простых суператомных булевых алгебр с выделенными плотными подалгебрами ширины $n$, элементарные теории которых различны и не имеют счетно-насыщенных моделей.

ДокАЗАТЕльство. Докажем, что модель $\mathfrak{A}_{I}$, построенная при доказательстве теоремы 2.4, простая. Пусть $\mathfrak{A}_{I}=\sum_{i \in I} \mathfrak{N}_{i}$. Напомним, что $T_{i} \# T_{j}$ для любых различных $i, j \in I$.

Несложно доказать, что $N\left(\mathfrak{A}_{I}\right)=\bigcup_{i \in I} N\left(\mathfrak{A}_{i}\right)$. Последнее влечет, что для всех $l \in N\left(\mathfrak{A}_{I}\right)$ будет $w_{\mathfrak{A}_{I}}(l)=1$. Таким образом, условия (а) и (б) из теоремы 3.4 выполняется тривиальным образом и, стало быть, модель $\mathfrak{A}_{I}$ простая.

Теорема доказана.

\section{ЛИТЕРАТУРА}

1. Кейслер Г., Чэн Ч. Теория моделей. М.: Мир, 1977.

2. Гончаров С. С. Счетные булевы алгебры и разрешимость. Новосибирск: Наука, 1997.

3. Пальчунов Д. Е. О неразрешимости теорий булевых алгебр с выделенным идеалом // Алгебра и логика. 1986. Т. 25, № 3. С. 326-346.

4. Pal'chunov Д. E. Countably-categorical Boolean algebras with distinguished ideals // Studia Logica. 1987. V. XLVI, N 2. P. 121-135.

5. Пальчунов Д. Е. Конечно-аксиоматизируемые булевы алгебры с выделенными идеалами // Алгебра и логика. 1987. Т. 26, № 4. С. 435-455.

6. Пальчунов Д. Е. Прямые слагаемые булевых алгебр с выделенными идеалами // Алгебра и логика. 1992. Т. 31, № 5. С. 499-537.

7. Пальчунов Д. Е. Простые и счетно-насыщенные модели теории булевых алгебр с выделенными идеалами // Тр. Ин-та математики СО РАН. 1993. Т. 25. С. 82-103.

8. Пальчунов Д. Е. Теории булевых алгебр с выделенными идеалами, не имеющие простой модели // Тр. Ин-та математики СО РАН. 1993. Т. 25. С. 104-132.

9. Пальчунов Д. Е., Трофимов А. В., Турко А. И. Автоустойчивость булевых алгебр с выделенными идеалами относительно сильных конструктивизаций // Сиб. мат. журн. 2015. Т. 56, № 3. С. $617-628$.

10. Пальчунов Д. Е., Трофимов А. В. Локальные и неисчезающие суператомные булевы алгебры с выделенной плотной подалгеброй // Алгебра и логика. 2011. Т. 50, № 6. C. $822-847$.

Поступила в редакцию 10 марта 2020 г.

После доработки 6 апреля 2020 г.

Принята к публикации 8 апреля 2020 г.

Пальчунов Дмитрий Евгеньевич, Трофимов Алексей Викторович

Институт математики им. С. Л. Соболева СО РАН,

пр. Академика Коптюга, 4, Новосибирск 630090;

Новосибирский государственный университет,

ул. Пирогова, 1, Новосибирск 630090

palch@math.nsc.ru, tr0f@mail.ru 